"Récits cinétiques »: le déplacement comme récit de contestation des wahayu, concubines de statut servile dans les régions frontalières du Niger et $d u$ Nigeria

'Kinetic Narratives': fugitive displacement as a narrative of protest by wahayu, concubine women of slave status in the borderlands of Niger and Nigeria "Relatos cinéticos": el desplazamiento como relato de protesta de las wahayu, concubinas de condición servil en las zonas fronterizas de Níger y de Nigeria "Relatos cinéticos »: a deslocação como relato de contestação das wahayu, concubinas de condição servil, nas zonas fronteiriças do Níger e da Nigéria

\title{
Lotte Pelckmans
}

Traducteur : Alice Beriot

\section{(2) OpenEdition} Journals

Édition électronique

URL : https://journals.openedition.org/slaveries/4469

DOI : $10.4000 /$ slaveries.4469

ISSN : 2540-6647

Éditeur

CIRESC

Référence électronique

Lotte Pelckmans, « «Récits cinétiques » : le déplacement comme récit de contestation des wahayu, concubines de statut servile dans les régions frontalières du Niger et du Nigeria », Esclavages \& Postesclavages [En ligne], 4 | 2021, mis en ligne le 10 mai 2021, consulté le 06 janvier 2023. URL : http:// journals.openedition.org/slaveries/4469; DOI : https://doi.org/10.4000/slaveries.4469

Ce document a été généré automatiquement le 6 janvier 2023.

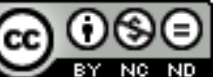

Creative Commons - Attribution - Pas d'Utilisation Commerciale - Pas de Modification 4.0 International - CC BY-NC-ND 4.0

https://creativecommons.org/licenses/by-nc-nd/4.0/ 
« Récits cinétiques » : le déplacement comme récit de contestation des wahayu, concubines de statut servile dans les régions frontalières du Niger et $d u$ Nigeria

'Kinetic Narratives': fugitive displacement as a narrative of protest by wahayu, concubine women of slave status in the borderlands of Niger and Nigeria "Relatos cinéticos": el desplazamiento como relato de protesta de las wahayu, concubinas de condición servil en las zonas fronterizas de Níger y de Nigeria "Relatos cinéticos »: a deslocação como relato de contestação das wahayu, concubinas de condição servil, nas zonas fronteiriças do Níger e da Nigéria

Lotte Pelckmans

Traduction : Alice Beriot

\section{Introduction : une lacune des récits écrits par des femmes esclaves?}

1 Depuis plusieurs années, je nourris l'espoir de découvrir des documents écrits par des individus au statut héréditaire d'esclave, et classifié comme tel, dans le Sahel francophone, qui seraient basés sur des vécus personnels d'exploitation, d'émancipation et de cohabitation. De tels matériaux sont difficiles à trouver, que ce soit dans les langues vernaculaires ou en français. Le contraste est immense avec le paradigme de l'esclavage atlantique. En effet, comme cela est analysé dans l'introduction de ce numéro, il y a pléthore de récits d'esclaves, de «nouveaux récits 
d'esclaves» (neo-slave narratives) et de romans portant sur l'esclavage atlantique. La disparité est donc grande avec le contexte post-esclavage francophone en Afrique de l'Ouest : rares sont les témoignages et les formes écrites de nature (auto)biographique transmis par ou pour le compte de groupes historiquement subordonnés.

2 Les quelques ouvrages existants sont généralement écrits par des hommes ouestafricains immigrés en Europe ou ailleurs, qui évoquent leur statut classificatoire d'esclave ou l'héritage d'un passé d'esclave dans le cadre de leurs mémoires. Autobiographique et issu de la diaspora, ce mince corpus littéraire donne donc uniquement à entendre des voix masculines. Les quelques récits écrits à propos de femmes ayant un statut classificatoire d'esclave ont été produits par des hommes militant à la tête de mouvements citoyens anti-esclavage (Abdelkader \& Zangaou 2012 pour Timidria; Temedt Association 2014) ou universitaires (Keita 2012). À ma connaissance, il n'existe aucune autobiographie écrite par une femme au statut d'esclave au Sahel.

3 Cette lacune est le fait d'une multiplicité de facteurs inhérents au Sahel postesclavagiste, liés entre autres au genre, à la classe sociale, à l'éducation et à l'origine ethnique. Au Sahel, les femmes dotées d'un statut héréditaire d'esclave font depuis longtemps partie des populations les plus pauvres; elles sont exclues du domaine de l'éducation, et connaissent des taux d'analphabétisme élevés, ce qui a considérablement limité leur visibilité.

4 Parmi les potentiels obstacles à l'écriture ou au témoignage de ces femmes, on trouve notamment des attitudes culturelles ambiguës par rapport au rôle et à la visibilité des femmes dans l'opinion publique. S'ajoute à cela une conception fortement ancrée du secret et du tabou qui entourent certains pans de l'héritage de l'esclavage par ascendance. De plus, des difficultés logistiques se posent pour ceux qui souhaiteraient recueillir les témoignages, telles que l'éloignement géographique, la pauvreté, la religion ou l'insécurité de la région. Cette absence notable de récits féminins portant sur les femmes au statut classificatoire d'esclave est encore accentuée par la pratique de la concubine qu'est la wahaya, phénomène au cœur de cet article.

\section{La pratique de la wahaya}

5 Les wahayu - wahaya au singulier - sont des femmes ayant un statut d'esclave qui sont prises comme concubines ${ }^{1}$ par des hommes libres, appelés " époux/maîtres ${ }^{2}$ » suivant le droit islamique (Gausset 2007). Selon les lois abolissant l'esclavage, cette pratique aurait dû être abandonnée, mais c'est loin d'être le cas. Lovejoy $(1988: 245)$ note : «Dans le califat de Sokoto, au nord du Nigeria, les pouvoirs de l'aristocratie étaient accrus par l'administration coloniale, qui tolérait le statu quo entourant la pratique de la wahaya ", "les concubines et les femmes esclaves étaient encore plus défavorisées que les femmes libres. Pour les Britanniques, la condition de la femme n'était pas une question importante. [...] Ce "concubinage" est donc resté autorisé ${ }^{3}$ " bien après l'abolition coloniale de l'esclavage domestique de 1907 au Nigeria.

6 Dans les travaux universitaires, la pratique de la wahaya au Sahel a été décrite au moyen de concepts décrivant une situation conjugale : "concubinage ", "esclavage conjugal », «foyer avec harem» (Mack 1990; Cooper 1994; Gausset 2007; Sinderud 2013 ; Jeppie, Moosa \& Roberts 2010 ; Rossi 2015), mariage «forcé » ou «secret » 
(McDougall 2016:226). Les organisations de défense des droits humains et les ONG parlent généralement d'«enlèvement», de «traite d'êtres humains", de "prostitution » ou d'« esclavage sexuel » (Dottridge 2005) pour décrire certains aspects de cette pratique. Un rapport de l'Organisation internationale pour les migrations (OIM) et de l'UN Refugee Agency (UNHCR), analysant les questions de nationalité, de migration et d'apatridie en Afrique de l'Ouest (Manby $2015: 79$ ), décrit le «système de wahaya " comme promouvant des "épouses supplémentaires» au nombre légal de quatre, en mariant des «filles d'origine "servile" ».

7 Dans les langues vernaculaires locales, différents termes sont utilisés en fonction du contexte et de la position du locuteur. Le terme arabe umm al-walad fait référence aux prescriptions juridiques des textes coraniques et désigne la femme esclave qui porte un enfant de son maître et qui sera affranchie à un moment ou à un autre de sa vie (à la naissance de l'enfant ou à la mort du maître) (Brockopp 2000:192-203). En langue haoussa, la lingua franca des régions frontalières reliant le Niger et le Nigeria, une femme ayant ce statut est appelée trivialement "sa daka», un terme qui, selon le rapport de Timidria, signifie littéralement "mettre dans sa chambre" ou encore "pousser dans la chambre», illustrant l'utilisation sexuelle de la femme concernée (Abdelkader \& Zangaou 2012:5). L'institution de la wahaya est connotée de diverses manières et perçue différemment par les habitants d'un Sahel principalement musulman. Bien que certains courants de l'idéologie islamique décrivent la pratique de la wahaya comme la possibilité la plus honorable et la plus digne pour une femme esclave de se marier, et comme une voie légale vers l'émancipation pour les femmes elles-mêmes ou, tout au moins, pour leurs enfants, la réalité ne semble pas toujours conforme à cet idéal.

8 L'ambiguïté conceptuelle et idéologique autour de cette pratique empêche la possibilité de représentations directes et sans équivoque des femmes concernées. S'il existe plusieurs textes en adjami ou en arabe écrits par des hommes juristes musulmans qui présentent l'institution de la wahaya sur un ton tout à fait neutre, rares sont les entretiens et les échanges reproduits dans les travaux universitaires contemporains qui offrent une vision vernaculaire venue d'« en bas » (Bellagamba 2017) concernant cette pratique. Le point de vue subjectif de ces femmes est donc peu représenté dans les sources empiriques, qu'elles soient anciennes ou contemporaines ${ }^{4}$.

\section{Post-esclavage}

9 Depuis l'abolition de l'esclavage domestique en 1905 dans les colonies françaises en Afrique, et en 1907 pour le Nigeria (alors colonie britannique), la wahaya est supposée avoir disparu. Si la pratique est clairement illégale d'après le droit constitutionnel national et selon les lois internationales, des visions mêlant conceptions coutumières et pensée islamique continuent de promouvoir cette tradition dans certaines régions. En effet, un grand nombre d'individus, issus de certaines régions et populations du Sahel, se voient toujours attribuer un statut d'esclave et peuvent être stigmatisés et exclus de diverses dimensions centrales de la vie sociale. Et comme il est quasi impossible d'échapper à ce statut, qui est transféré d'une génération à une autre, les personnes d'ascendance servile demeurent nombreuses. Leur statut est héréditaire et elles appartiennent à une catégorie transmise de manière quasi perpétuelle par les parents 
et la lignée (Thioub 2012). Il en résulte un «esclavage classificatoire » (Rossi $2009: 4$ ), aussi appelé « esclavage par ascendance ».

10 Au regard de la complexité de ce contexte post-esclavagiste mêlant idéologies et affects relatifs à cette pratique, comment accéder au vécu subjectif des femmes elles-mêmes concernant leur situation, leur statut et leurs aspirations passées et futures, pour elles et pour leurs familles? Ce questionnement est à l'origine du postulat de cet article, qui s'appuie sur une forme alternative d'expression narrative : le mouvement, la cinétique ${ }^{5}$. Je considère que les femmes dont il est question dans ce texte s'engagent dans un déplacement fugitif, aussi appelé «marronnage » dans le contexte transatlantique et, ce faisant, nous racontent l'histoire d'une rupture avec des normes sociales. Leur fuite, leur marronnage véhiculent le message selon lequel la pratique de la wahaya est inacceptable pour la femme qui la subit. Indépendamment des points de vue émis par les militants, des autres concubines ou de l'idéologie islamique, ce sont ces mouvements de fugue qui expriment la manière dont les femmes vivent subjectivement leur condition de concubine.

\section{Biais des sources et aperçu fractal des narrations}

11 Faute de témoignages directs de trajectoires féminines individuelles, j'ai été amenée à me fonder sur une seule source : un rapport issu d'entretiens conduits avec des wahayu par une organisation anti-esclavagiste basée au Niger, portant le nom de Timidria (Alou 1996) et cofinancée par Anti-Slavery International et par le Department for International Development (DFID), deux organisations basées au Royaume-Uni. Ce rapport - selon les descriptions de l'organisation elle-même - a sélectionné neuf cas extraits d'un corpus de 165 entretiens, principalement avec des wahayu et ex-wahayu au Niger (région de Tahoua) et au Nigeria (États de Kano, Sokoto, Kaduna et Zaria) (Abdelkader \& Zangaou [ci-après A \& Z] 2012).

12 Les biais introduits par le choix de cette source sont importants. Premièrement, ces entretiens concernent pour la plupart des femmes qui sont sorties du système par la fuite ; deuxièmement, je n'ai pas pu contrôler ni assister à la production des données sur lesquelles se fonde ce travail. Pour souligner la médiation entourant les narrations qui, on peut le supposer, ne donnent qu'un aperçu fractal sur les réalités vécues par ces femmes, j'utilise le terme de « récits " (Eastmond 2007) plutôt que « témoignages », tel qu'employé dans le rapport de Timidria. Pour moi, des témoignages doivent concerner une matière brute, source directe, idéalement sans intermédiaire. Vu que les écrits en question proviennent d'entretiens avec des femmes, ils sont indirects: le terme "récits» correspond donc mieux. Mon article n'a pas pour objectif de quantifier exactement le nombre de femmes ayant un statut de concubine (entreprise impossible dans tous les cas), ni de retranscrire des témoignages détaillés : il vise à illustrer et à mettre l'accent sur les mouvements de fuite, réels ou souhaités, de certaines concubines interrogées.

13 Même si les conditions d'élaboration de ce rapport sont hors de toute appréciation critique, je souhaite argumenter à partir de ce corpus, aussi restreint soit-il. Les récits sont à recontextualiser à partir de recherches historiques sur le marronnage d'hommes et de femmes esclaves au Sahel (Rossi 2017 ; Rodet 2009) et à combiner aux entretiens que j'ai moi-même menés avec des femmes concubines au Mali central. Ces derniers contiennent beaucoup de mentions et de références à d'autres wahayu qui ont 
également pris la fuite. Partant de là, je pose comme hypothèse que la pratique de la fuite, du marronnage est importante dans les zones transfrontalières du sud du Niger et du nord du Nigeria, au-delà des neuf récits enregistrés dans ce rapport. Ces régions se caractérisent par une forte perméabilité et par des interactions historiques entre commerçants, nomades et autres acteurs ${ }^{6}$. Dans le califat de Sokoto (1804-1903), la circulation des femmes était intense ; les femmes esclaves étaient offertes en cadeau et circulaient entre suzerains et vassaux, voyageurs ou invités. Ainsi, ces régions représentent de longue date un espace crucial $^{7}$ dans les nombreuses migrations saisonnières et le nomadisme pastoral. La vulnérabilité et la pauvreté y sont généralisées, surtout pour les femmes et leurs familles ayant un statut d'esclave. Il est fréquent que leurs parents demeurent pour toujours dans l'ignorance quant au traitement réservé à leur fille et à sa localisation après son mariage comme concubine.

\section{Le marronnage / la fuite comme récit cinétique de dissidence}

14 Si les wahayu ont été initialement déplacées du Niger vers des foyers du nord du Nigeria, je m'intéresse ici aux mouvements transfrontaliers inverses, à savoir ceux impliquant les fuites de wahayu du Nord-Nigeria vers le Niger. Focalisons-nous donc sur une forme spécifique de récit cinétique : la fuite par laquelle une femme wahaya quitte le domicile de son ex-époux/maître, déployant ainsi une stratégie de résistance exprimant sa dissidence.

\section{Fuir}

Le rapport de Timidria identifie plusieurs éléments à l'origine du désir de fuite des wahayu: tout d'abord, les ventes et changements d'époux/maitre à répétition, découlant $d u$ fait que ces femmes peuvent être cédées, revendues ou rachetées à tout moment, notamment lorsqu'elles n'ont pas encore eu d'enfant. Ensuite, il y a la pénibilité du travail physique, les humiliations et les insultes basées sur leur statut ou celui de leurs enfants, la jalousie et les mauvais traitements des épouses officielles; et enfin, le fort sentiment d'aliénation dû à la rupture avec la famille, la langue et le foyer de ces femmes.

Du fait de ces épreuves, nombre de concubines interrogées dans le cadre de ce rapport ont exprimé leur volonté de fuir ; mais seules quelques-unes sont passées à l'acte. Il n'est pas toujours possible de réaliser son désir de fuite, comme le déclarait une wahaya vivant toujours avec son époux/maître : "Je partirai dès que l'opportunité se présentera. Nous sommes de véritables prisonnières. » (A \& Z 2012 : 22). Tabas Aborak raconte quant à elle (A \& Z $2012: 13$ ) :

Je devais m'occuper des enfants des maîtresses et des quatre wahayu. [...] Je redoutais leurs voyages, car c'était à moi de leur préparer un cheval, et je devais suivre à pied, porter l'enfant qu'elles allaitaient sur mon dos, à l'aller et au retour. Cela m'a toujours donné envie de fuir. [...]

17 Tadant, wahaya elle aussi demeurée chez son époux/maître, évoque les conséquences de la fuite d'une de ses semblables (A \& Z $2012: 23$ ) :

Il y avait une autre wahaya [...] abusée par le maître, plus tard elle a fui chez ses parents. [...] Avant la fuite de Tantout, les trois wahayu que nous sommes, vivions 
ensemble dans une même pièce, mais maintenant nous avons chacune notre chambre. Les chambres des épouses officielles sont décorées jusqu'au plafond, avec des lits et d'autres meubles. La mienne a juste un vieux matelas sale. point la manière dont elle et ses enfants étaient traités, était insupportable. Elle souligne que le plus intolérable était son incapacité à jouer son rôle de mère (A \& $\mathrm{Z}$ $2012: 15)$ :

C'était devenu insupportable, pour mes enfants et moi. Ils avaient besoin de ma protection et de mon affection, mais tout ce qu'ils recevaient, c'était ce genre de traitement. Je ne pouvais jamais les soutenir moralement, alors que je sais qu'ils en avaient besoin. Quand j'ai compris que mes enfants étaient de plus en plus conscients de mon incapacité à jouer mon rôle de mère pour eux, j'ai commencé à élaborer un plan pour nous sortir de cette situation qui empirait. Je voulais m'enfuir avec mes enfants. Mais c'était impossible. Alors, quand des Peuls sont arrivés du Niger avec leurs troupeaux, j'ai saisi l'opportunité de fuir, en abandonnant mes enfants.

La plupart des wahayu indiquent avoir fui sans avoir pu emmener leurs enfants et avec comme unique point de repère le seul nom de Zongon Ablo. Tabas Aborak, l'une d'entre elles, note à son propos (A \& Z 2012 : 13) :

J'ai passé des jours et des nuits dans l'angoisse la plus totale, traversant des villages et hameaux en demandant si les gens savaient où vivaient les Touareg noirs. Je ne me rappelle pas de tous les villages par lesquels je suis passée, mais je me souviens du nom du village de Zongon Ablo.

Ces éléments centraux varient d'un cas à l'autre, mais le caractère incertain de l'évasion constitue un trait commun dans un grand nombre de ces récits.

\section{Zongon Ablo : un refuge ou un village de marronnes}

Sur les cinq femmes wahayu ayant fui et "témoignant " dans le rapport, trois vivent actuellement à Zonglo Ablo dans le département de Birni N'Konni au Niger. Tabas Aborak, l'une d'entre elles, note à son propos (A \& Z 2012 : 13) :

Zongon Ablo est un village dont $80 \%$ des habitants, principalement les femmes, sont originaires d'autres endroits. J'ai tout fait pour y arriver. Je me souviens être passée par Guidan Iddar. Zongon Ablo ! J'étais enfin arrivée.

Elle ajoute qu'il se trouve dans la zone des «Touaregs noirs ", terme indiquant souvent le statut d'esclave. D'autres femmes, particulièrement celles ayant fui le Nigeria pour le Niger, se sont réfugiées dans ce village où résident un grand nombre d'ex-wahayu ayant fui le domicile de leur maître. Dans ces sociétés très patriarcales où les femmes sont supposées être protégées par le mariage, tenter de gagner sa vie en ville constitue une autre option pour ces femmes en fuite qui ne peuvent revenir dans leur foyer : la plupart des ex-wahayu sont alors contraintes de s'y prostituer (A \& Z 2012 : 7). Cette 
situation est très probablement la conséquence d'une absence totale de protection sociale en cas de retour à une vie non maritale.

Aussi Zongon Ablo est-il devenu un nouveau lieu d'appartenance et de connexion, un asile pour toutes celles qui n'ont plus de foyer à elles. Par de nombreux aspects, il s'agit d'une sorte de village pionnier, situé aux confins d'un cadre sociétal qui façonne les notions d'appartenance et d'identité sur la base des hiérarchies sociales héritées du passé esclavagiste.

\section{La famille : un refuge incertain}

Un autre obstacle à la fuite est que les femmes étaient parfois si jeunes quand elles ont été vendues et acquises, qu'elles ne savent même pas d'où elles viennent ou n'ont qu'un vague souvenir de leur foyer et n'ont en mémoire que le nom de leurs parents. La wahaya Tabalole Bouweye s'est échappée du domicile de son troisième époux/maître pour revenir chez le premier, car il s'agissait du seul endroit qui avait pour elle représenté un foyer (A \& Z $2012: 24$ ) :

À cette époque, il y avait trois autres wahayu, [...] qui ont toutes fini par s'échapper.

J'ai aussi fini par m'échapper il y a 17 ans. Je suis repartie vivre avec feu Aillale III de Sokoto [mon premier maître], dans le seul foyer que je connaissais, parce que j'avais été élevée là-bas. Je n'ai pas connu ma famille. J'ai été vendue jeune.

Certaines femmes ont été vendues avant d'être réellement considérées comme des concubines. Plusieurs d'entre elles commencent dans un foyer comme domestiques, avant d'être finalement cédées ou vendues à un homme en tant que concubines. Celles qui se souviennent de leur famille et qui continuent d'être en contact avec elle peuvent choisir de rentrer chez leurs parents mais généralement, d'après le rapport de Timidria, ces derniers les renvoient alors d'où elles viennent, dans la mesure où elles appartiennent officiellement à leur "propriétaire». Il y a toujours des exceptions : toutes les femmes n'ont pas le même destin. Ainsi, les wahayu bénéficiant d'un plus grand soutien de leurs familles peuvent revenir dans leur foyer, notamment parce qu'en acceptant le «mariage » de leur fille, ces familles n'ont pas saisi à quel point la culture haoussa de certaines parties du Nigeria septentrional peut être différente de la leur, particulièrement en ce qui concerne la pratique de la wahaya. Par exemple, les familles peuvent juger inacceptable l'interdiction qui leur est faite de rendre visite à leur fille.

\section{Dissidence}

L'histoire d'Akarat Idimi illustre la fierté de certaines femmes et leur capacité à résister aux épreuves (A \& Z 2012 : 20) :

Mes parents ont compris les abus que ma famille et moi avions subis dans la maison de Sarkin Koudou [l'époux/maître] et ne m'ont pas renvoyée chez lui. Mon soidisant «mari » comptait sur mon maître pour me ramener, mais celui-ci n'avait plus aucun pouvoir sur moi, puisqu'il m'avait vendue. Je n'avais plus à lui rendre de comptes. C'est ce que les Haoussa de Sokoto ne comprenaient pas. Selon eux, l'esclave fait partie d'un cheptel humain dans lequel le maître peut puiser autant qu'il veut. C'est comme ça que mon soi-disant mariage s'est terminé, parce que j'ai refusé catégoriquement de revenir. 
Il faut noter que, dans ce récit, Akarat évoque certaines différences d'ordre culturel dans les régions frontalières entre le Niger et le Nigeria: "ce sont les Haoussa de Sokoto qui ne comprenaient pas ce qu'est un véritable mariage, ils trompent les gens et leur font croire qu'il s'agit d'un mariage.» (ibid.). Par ce discours, Akarat confirme que sa fuite et son refus de revenir auprès de son époux/maître est une manière de s'opposer aux souffrances intolérables et au traitement qui lui étaient réservés " comme un animal dans un troupeau », et non comme un être humain ayant un statut de concubine. Par ailleurs, il est intéressant de noter qu'elle ne raisonne pas en termes juridiques contemporains, mais s'appuie sur une logique qui valide le point de vue des maitres, selon lequel l'esclavage serait légal. En d'autres termes, elle ne dit pas qu'il est illégal pour un maître d'avoir eu du pouvoir sur elle : elle réaffirme au contraire la légalité de sa vente qui fait que son maître n'a plus de droit sur elle. Ce faisant, Akarat illustre clairement le pluralisme juridique pratiqué dans ces zones où le droit étatique est souvent méconnu et où certains courants du droit islamique sont favorisés.

\section{Conclusion}

Les formes écrites que sont l'(auto)biographie et le témoignage, transmis par ou au nom de groupes historiquement dominés en Afrique occidentale francophone, restent très peu répandues. Le corpus extrêmement mince de textes autobiographiques et issus de la diaspora traitant du contexte post-esclavagiste, est principalement le fait de voix masculines.

31 Face à cette sous-représentation, à l'absence d'autorité narrative et à la nature fractale de la narration des rares témoignages de concubines esclaves enregistrés en Afrique occidentale - eux-mêmes réalisés dans le cadre d'un rapport contre l'esclavage -, j'ai analysé une forme narrative alternative, distincte des modes écrits, visuels ou oraux qui constituent les « récits » tels que nous les entendons.

32 Le concept de "récit cinétique " permet de saisir de quelle manière le mouvement physique nous renseigne sur le discours propre de ces femmes quant à leurs expériences, à leurs points de vue et à leurs aspirations à une existence digne. La mobilité en tant que stratégie d'émancipation des esclaves a fait l'objet de nombreuses recherches ${ }^{8}$, mais peu de travaux se sont penchés sur le cas de ces femmes toujours définies - aujourd'hui encore - par leur statut classificatoire d'esclave et de concubine.

Par la mobilité fugitive qu'ils décrivent, les récits des wahayu manifestent l'aspiration de ces femmes à la liberté et à la respectabilité en matière de relations familiales, par le biais d'un mariage et d'un statut de mère socialement reconnu. Ce mouvement du corps en fuite témoigne de souffrances prolongées, que ces concubines de statut servile continuent de subir à ce jour. Ces femmes qui fuient les épreuves endurées dans le foyer de leur époux/maître, le font en dépit de normes sociétales et religieuses fortement ancrées. La nature patriarcale du système colonial, du monde académique et de la culture, et son impact dans l'histoire de la région, ont souvent occulté la place des femmes, tant dans les discours que dans les actions et narrations. Enfin, les visions et solutions humanitaires apportées face aux souffrances des personnes économiquement défavorisées et présentant un statut d'esclave émanent des conceptions occidentales, individualistes et libérales de la liberté, étayées par une vision universelle des droits humains. Ce positionnement continue d'éclipser et d'invisibiliser d'autres voix et 
formes d'émancipation plus subtiles, telles que les fuites d'esclaves parmi les Africains, et particulièrement les Africaines. Pour la plupart des femmes vivant au Sahel, la liberté est conditionnée par la reconnaissance sociale de leur rôle de femme et d'épouse, et non par le fait de devenir un individu disposant d'une totale liberté de mouvement. Néanmoins, c'est paradoxalement par la mise à mal de cette liberté de relation et d'appartenance que la fuite et la capacité individuelle à se mouvoir deviennent pour certaines femmes le seul moyen de sortir de leur condition.

Pour finir, je souhaiterais attirer l'attention du lecteur sur le fait que cet article a certes mis l'accent sur les fuites de femmes qui vivent la pratique de la wahaya comme une forme d'exploitation non désirable: néanmoins, nombre d'entre elles restent concubines. Mon choix de me focaliser sur les récits cinétiques et sur la mobilité des fugitives n'a pas vocation à oblitérer les expériences de concubines ayant potentiellement généré des situations heureuses et/ou des opportunités d'ascension sociale.

\section{BIBLIOGRAPHIE}

ABDELKADER, Galy Kadir \& Moussa ZANGAOU, 2012. WAHAYA: Domestic and sexual slavery in Niger, rapport établi par l'association Timidria, en collaboration avec Anti-Slavery International. Disponible en ligne : http://www.antislavery.org/wp-content/uploads/2018/10/Wahayareport.pdf [dernier accès, avril 2021].

Alou, Mahaman Tidjani, 1996. « Les Trajectoires d'une Reconversion du Militantisme Associatif au Courtage en Développement: Le Cas de Timidria au Niger ", Bulletin de l'APAD, $\mathrm{n}^{\circ} 12$. Disponible en ligne : https://journals.openedition.org/apad/601 [dernier accès, avril 2021].

BellagamBA, Alice, 2017. « Freedom from Below. Some Introductory Thoughts ", Journal of Global Slavery, $\mathrm{n}^{\circ}$ 2/1-2, p. 1-9. Disponible en ligne : https://doi.org/10.1163/2405836X-00201013 [dernier accès, avril 2021].

BROCKOPP, Jonathan E., 2000. Early Mālikī Law: Ibn 'Abd Al-Hakam and His Major Compendium of Jurisprudence, Leiden, Brill.

COOPER, Barbara M., 1994. «Reflections On Slavery, Seclusion And Female Labor in the Maradi Region of Niger in the Nineteenth And Twentieth Centuries ", The Journal of African History, $\mathrm{n}^{\circ}$ 35/1, p. 61-78. Disponible en ligne : https://doi.org/10.1017/S0021853700025962 [dernier accès, avril 2021].

DOTTRIDGE, Mike, 2005. « Types Of Forced Labour And Slavery-Like Abuse Occurring In Africa Today ", Cahiers d'Études africaines, $\mathrm{n}^{\circ} 45, \mathrm{n}^{\circ}$ 179-180, p. 689-712. Disponible en ligne : https:// doi.org/10.4000/etudesafricaines.14968 [dernier accès, avril 2021].

EASTMOND, Marita, 2007. « Stories as Lived Experience: Narratives in Forced Migration Research », Journal of Refugee Studies, $\mathrm{n}^{\circ}$ 20/2, p. 248-264. Disponible en ligne : https://doi.org/10.1093/jrs/ fem007 [dernier accès, avril 2021]. 
EL HAMEL, Chouki, 2013. Black Morocco: A History of slavery, race and Islam, Cambridge, Cambridge University Press.

GAUSSET, Quentin, 2007. «Women, Gender and Slavery: Sub-Saharan Africa », dans Joseph Suad (dir.), Encyclopedia Of Women And Islamic Cultures: Economics, Education, Mobility And Space, Leiden / Boston, Brill, vol. 4, p. 510-513.

JEPPIE, Shamil, Ebrahim MOOSA \& Richard L. ROBERTS, éd., 2010. Muslim Family Law in Sub-Saharan Africa: Colonial Legacies and Post-colonial Challenges, Amsterdam, Amsterdam University Press.

KEITA, Naffet, dir., 2012. L'esclavage au Mali, Paris, L'Harmattan.

KLEIN, Martin A. \& Richard ROBERTS, 1980. « The Banamba Slave Exodus of 1905 and the Decline of Slavery in the Western Sudan », The Journal of African History, n² 21/3, p. 375-394.

LOVEJoY, Paul E., 1988. « Concubinage and the Status of Women Slaves In Early Colonial Northern Nigeria ", The Journal of African History, $n^{\circ} 29 / 2$, p. 245-266. Disponible en ligne : https://doi.org/ $10.1017 /$ S0021853700023665 [dernier accès, avril 2021].

LOVEJOY, Paul E., 1990. « Concubinage in the Sokoto caliphate (1804-1903) », Slavery \& Abolition, $\mathrm{n}^{\circ}$ 11/2, p. 159-189. Disponible en ligne : https://doi.org/10.1080/01440399008575005 [dernier accès, avril 2021].

LYDON, Gislaine, 2007. « Islamic Legal Culture And Slave-Ownership Contests In Nineteenth Century Sahara », International Journal of African Historical Studies, $n^{\circ} 40 / 3$, p. 391-439.

MACK, Beverly B., 1990. « Service and Status: Slaves and Concubines in Kano, Nigeria ", dans Roger Sanjek \& Shellee Colen, At Work in Homes: Household Workers in World Perspective, Washington, D.C., American Anthropological Association, p. 14-34.

MANBY, Bronwen, 2015. Nationality, Migration and Statelessness in West Africa: A Study for UNHCR and IOM. Disponible en ligne : https://www.unhcr.org/ecowas2015/Nationality-Migration-andStatelessness-in-West-Africa-REPORT-EN.pdf [dernier accès, avril 2021].

MCDOUGALL, E. Ann, 1998. « A Sense Of Self: The Life Of Fatma Barka », Canadian Journal of African Studies, $\mathrm{n}^{\circ}$ 32/2, p. 285-315. Disponible en ligne : https://doi.org/10.2307/486151 [dernier accès, avril 2021].

MCDOUGALL, E. Ann, 2016. « Concubinage as Forced Marriage?

Colonial jawari, contemporary hartaniyya and marriage in the Islamic Republic of Mauritania », dans Annie Bunting, Benjamin N. Lawrance \& Richard L. Roberts, éd., Marriage by Force? Contestation over Consent and Coercion in Africa, Ohio, Ohio University Press, p. 220-245.

NAST, Heidi, 2005. Concubines and Power: Five hundred years in a Northern Nigerian Palace, Minneapolis, University of Minnesota Press.

RODET, Marie, 2009. Les migrantes ignorées du Haut-Sénégal (1900-1946), Paris, Karthala.

RODET, Marie, 2011. "Sexualité, mariage et esclavage au Soudan français à la fin du XIXe siècle ", Clio. Femmes, genre, histoire, $\mathrm{n}^{\circ}$ 33, p. 45-64. Disponible en ligne : https://journals.openedition.org/ clio/10009 [dernier accès, avril 2021].

ROSSI, Benedetta, 2009. Reconfiguring Slavery: West African Trajectories, Liverpool, Liverpool University Press.

ROSSI, Benedetta, 2015. « African Post-Slavery: A History of the Future », The International Journal of African Historical Studies, $\mathrm{n}^{\circ} 48 / 2$, p. 303-324. 
RossI, Benedetta, 2017. « Freedom Under Scrutiny », Journal of Global Slavery, nº 1-2, p. 185-194. Disponible en ligne : https://doi.org/10.1163/2405836X-00201014 [dernier accès, avril 2021].

SINDERUD, Marte B., 2013. « Royal Concubinage in Ngaoundere, Northern Cameroon, ca. 19001960 », The International Journal of African Historical Studies, n 46/1, p. 1-25.

TEMEDT Association, 2014. Esclavage Au Mali. Des Victimes Témoignent, Paris, L'Harmattan.

THIOUB, Ibrahima, 2012. « Stigmas and Memory of Slavery In West Africa: Skin Color and Blood as Social Fracture Lines ", New Global Studies, $n^{\circ}$ 6/3. Disponible en ligne : https://doi.org/ 10.1515/1940-0004.1188 [dernier accès, avril 2021].

\section{NOTES}

1. J'ai choisi le terme "concubine», qui traduit probablement le mieux le terme d'origine zarma (Niger) wahaya (pl. wahayu). La concubine désigne ici une femme faisant l'objet d'une forme de "mariage " alternative, impliquant un ensemble de rituels, d'attentes et de modalités distinct de celui véhiculé par un mariage traditionnel dans les communautés concernées.

2. J'utilise le terme "époux/maître " afin de différencier ce statut de celui d'époux officiel, dans la mesure où la transaction permettant d'acquérir une concubine est considérée comme un paiement, qui offre à l'acheteur tous les droits de propriété sur la femme en question. Plus qu'un époux, ce personnage masculin est avant tout le propriétaire d'une concubine.

3. [N.D.A. : les citations en français de sources en langue étrangère sont des traductions de ma part.] Par ailleurs, comme l'ont démontré Marie Rodet (2011) et d'autres auteur $\cdot e \cdot s$, la possession de concubines était également une pratique dans les colonies françaises au Sahel parmi les officiers coloniaux eux-mêmes.

4. Notons quelques exceptions: Mack 1990; Lovejoy 1988, 1990 ; McDougall 1998; Lydon 2007; Sinderud 2013 - ces auteurs ayant retranscrit des témoignages de concubines ouest-africaines au milieu et à la fin du $\mathrm{xx}^{\mathrm{e}}$ siècle.

5. Sur le site Internet de l'Encyclopcedia britanica, le concept de « cinétique » est décrit de la manière suivante : « branche de la mécanique classique analysant l'effet des forces et couples sur le mouvement de corps dotés d'une masse » (www.britannica.com/science/ kinetics; dernier accès avril 2021).

6. Il ne s'agit pas de sous-entendre que le statut de concubine existe uniquement dans les régions frontalières. Pour des travaux portant sur des pratiques à une échelle plus locale ou nationale, voir Cooper 1994 (Niger), Nast 2005 (Nigeria) et El Hamel 2013 (Maroc).

7. L'influence de ce contexte transnational, associée aux nouveaux cadres juridiques postcoloniaux, sur des questions ayant trait au mariage, à l'esclavage, au statut de concubine et à leurs implications légales, est un sujet qui s'inscrit au-delà de la portée du présent article; elle n'y sera donc pas développée. Notons simplement que ce contexte a pu contribuer à la controverse entourant les pratiques liées à la condition féminine telles que décrites ici. 
8. Pour ne nommer ne serait-ce qu'un seul exemple sur l'Afrique de l'Ouest, voir Klein \& Roberts 1980. Pour une analyse plus spécifique de cas de femmes fuyant leur condition d'esclave, voir Rodet 2009.

\section{RÉSUMÉS}

Que ce soit en langue vernaculaire et en français, il existe peu de récits écrits relatant des expériences subjectives d'exploitation, de liberté, d'émancipation et de cohabitation d'individus ayant le statut héréditaire d'esclaves au Sahel. Les quelques documents écrits traitant des femmes de cette catégorie ont été produits par des organisations de lutte contre l'esclavage, comme Timidria au Niger, Temedt au Mali, ou bien par des universitaires. Afin de rendre compte de l'expérience subjective de ces femmes, au-delà des rares témoignages oraux, souvent biaisés, recueillis par les mouvements anti-esclavagistes, je propose de sortir des sentiers battus des formes écrites, visuelles et orales qui abondent dans les sphères intellectuelles occidentales, et de me pencher sur une de leurs formes alternatives, composée de mouvements et de langage corporel : la cinétique.

Par « récits cinétiques », je fais référence à une im/mobilité physique agissante et incarnée qui traduit les expériences subjectives des femmes au statut héréditaire d'esclaves au Sahel. Plus spécifiquement, ce sont leurs déplacements fugitifs que j'analyse comme des tactiques de résistance à leur exploitation.

Cet article se fonde sur l'exemple spécifique des mouvements de fuite des concubines classées parmi les esclaves dans les régions frontalières du Niger et du Nigeria. Ces mouvements de femmes ressemblent aux marronnages des esclaves de l'océan Atlantique et de l'océan Indien. La source principale est un rapport basé sur des entretiens menés avec des concubines par une organisation anti-esclavagiste établie au Niger. Ce rapport a sélectionné neuf cas parmi un corpus de 165 entretiens, principalement dans le sud du Niger et le nord du Nigeria. Les biais introduits par le choix de cette source sont importants et nombreux, mais ils sont aussi contextualisés par les vingt ans d'expérience de l'auteure avec des cas similaires dans le centre du Mali.

Les wahayu - wahaya au singulier - sont des femmes au statut d'esclave qui sont prises comme concubines par des hommes libres, selon la loi islamique. Selon les lois abolissant l'esclavage, cette pratique aurait dû être abandonnée, mais c'est loin d'être le cas. Je développe la notion de post-esclavage pour expliquer en quoi l'abolition de l'esclavage n'a pas mis fin aux exclusions fondées sur le statut social (descendants de maitres versus descendants d'esclaves).

Depuis l'abolition de l'esclavage par les forces coloniales en Afrique au début du xx siècle, une ambiguïté juridique, conceptuelle et idéologique entoure la pratique de la wahaya : ceci est cause que le point de vue subjectif de ces femmes est peu représenté dans les sources. Pour y remédier, le présent article plaide pour que les mouvements cinétiques soient pris en considération comme des récits : dans ce cas précis, les déplacements fugitifs des wahayu captent les aspirations à la liberté et à la dignité de ces femmes concubines au statut servile dans le Sahel.

Les femmes du rapport mentionnent dans leurs récits comment elles ou d'autres wahayu ont fui. $\mathrm{Au}$ vu des multiples références à leurs mobilités et à celles des autres, je pose comme hypothèse que la pratique de la fuite, du marronnage individuel dans les zones transfrontalières du sud du Niger et du nord du Nigeria est significative et va bien au-delà des neuf récits enregistrés dans le rapport. L'article analyse plusieurs motifs qui sont à l'origine du désir de fuite des wahayu et 
illustre, par des exemples concrets tirés des récits, plusieurs stratégies et résultats de la fuite. En conclusion, les récits cinétiques, à savoir les fuites par lesquelles les femmes wahayu quittent la maison de leur mari/maître, sont analysés comme une tactique subalterne de résistance et de dissidence, et comme une aspiration à une existence digne.

Le mouvement du corps en fuite témoigne de la souffrance prolongée que ces concubines continuent d'endurer à ce jour. Certes, pour la plupart des femmes du Sahel, la liberté est conditionnée par la reconnaissance sociale de leur rôle de femme et d'épouse, et non par le fait de devenir des individus disposant d'une totale liberté de mouvement. Mais, paradoxalement, c'est précisément par la mise à mal de cette liberté de relation et d'appartenance que la fuite et la capacité individuelle de mouvement deviennent pour certaines la seule issue à leur condition.

In both the local vernacular and French, there are few written accounts of the subjective experiences of exploitation, freedom, emancipation, and cohabitation of individuals with the hereditary status of slaves in the Sahel. The few written documents dealing with women in this category were produced by anti-slavery organizations, such as Timidria in Niger, Temedt in Mali, or by academics. In order to shed some light on the subjective experiences of these women, beyond the rare and often biased oral testimonies collected by anti-slavery movements, I propose to go off the beaten track of the written, visual and oral forms that abound in Western intellectual spheres, and to look at one of the alternative forms, composed of movements and body language: kinetics.

By 'kinetic narratives', I refer to an embodied and active physical im/mobility that translates the subjective experiences of women with hereditary slave status in the Sahel. More specifically, it is their fugitive movements that I analyze as tactics of resistance to their exploitation.

This paper uses the specific example of the fugitive movements of concubines classified as slaves or descendants of slaves in the border regions of Niger and Nigeria. These movements of women resemble the marronage of slaves in the Atlantic and Indian Oceans. The main source is a report based on interviews with concubines conducted by a Niger-based anti-slavery organization with nine cases from a corpus of 165 interviews, mainly in southern Niger and northern Nigeria. The biases introduced by the choice of this source are significant and numerous, but they are also countered and contextualized by the author's twenty years of experience with similar cases in central Mali.

Wahayu - wahaya in the singular - are women with slave status who are taken as concubines by free men, according to Islamic law. According to the laws abolishing slavery, this practice should have been abandoned, but this is far from the case. I develop the notion of post-slavery to explain how the abolition of slavery did not end exclusions based on social status (descendants of masters versus slaves).

Since the abolition of slavery by colonial forces in Africa in the early 20th century, legal, conceptual, and ideological ambiguity surrounds the practice of wahaya: this is because the subjective views of these women are poorly represented in the sources. To remedy this, this paper argues that kinetic movements should be considered as narratives: in this case, the fugitive movements of the wahayu capture the aspirations for freedom and dignity of these concubine women with servile status in the Sahel.

The women in the report share in their stories how they or other wahayu fled. Given the multiple references to their own and others' mobilities, I hypothesize that the practice of flight, of marooning in the cross-border areas of southern Niger and northern Nigeria, is significant and goes well beyond the nine accounts recorded in the report. The paper analyzes several motives behind the wahayu desire to flee, and illustrates, through concrete examples from the narratives, several strategies and outcomes of flight.

In conclusion, the kinetic narratives, namely the escapes by which wahayu women leave the house of their husband/master, are analyzed as a subaltern tactic of resistance and dissent, and 
as an aspiration for a dignified existence.

The movement of the fleeing body testifies to the prolonged suffering that these concubines continue to endure to this day. Certainly, for most women in the Sahel, freedom is conditioned by social recognition of their role as women and wives, not by becoming individuals with complete freedom of movement. But, paradoxically, it is precisely through the undermining of this freedom of relationship and belonging that flight and individual capacity for movement become for some the only way out of their condition.

Tanto en lengua vernácula como en francés, existen pocos relatos escritos que cuenten experiencias subjetivas de explotación, de libertad, de emancipación y de cohabitación de individuos con el estatus hereditario de esclavos en el Sahel. Los pocos documentos escritos que tratan de mujeres de esta categoría fueron producidos por organizaciones de lucha contra la esclavitud, como Timidria, en Níger, Temedt en Mali, o por académicos. Para dar cuenta de la experiencia subjetiva de estas mujeres, más allá de los escasos testimonios orales, a menudo sesgados, tomados por los movimientos antiesclavistas, propongo abandonar los caminos trillados de las formas escritas, visuales y orales que abundan en las esferas intelectuales occidentales, abordando una de sus formas alternativas, compuesta de movimientos y de lenguaje corporal: la cinética.

Por "relatos cinéticos" entiendo una in/movilidad física actuante y encarnada que traduce las experiencias subjetivas de mujeres con el estatus hereditario de esclavas en el Sahel. Más específicamente, analizo sus desplazamientos fugitivos como tácticas de resistencia a la explotación.

Este artículo se basa en el ejemplo específico de los movimientos de fuga de las concubinas clasificadas como esclavas en las regiones fronterizas de Níger y de Nigeria. Estos movimientos de mujeres se asemejan a los cimarronajes de los esclavos del océano Atlántico y del océano Índico. La fuente principal es un informe basado en entrevistas a concubinas, llevadas a cabo por una organización antiesclavista radicada en Níger. Este informe ha seleccionado nueve casos entre un corpus de 165 entrevistas, principalmente en el sur de Níger y en el norte de Nigeria. Los sesgos introducidos por la elección de esta fuente son numerosos e importantes, pero también están contextualizados por los veinte años de experiencia del autor, con casos similares en el centro de Mali.

Las wahayu - wahaya en singular- son mujeres con estatus de esclavas, tomadas como concubinas por hombres libres, según la ley islámica. De acuerdo con las leyes de abolición de la esclavitud, esta práctica debería haber sido abandonada, pero dista mucho de ser el caso. Desarrollo la noción de post-esclavitud para explicar de qué modo la abolición de la esclavitud no puso fin a las exclusiones basadas en el estatus social (descendientes de amos versus descendientes de esclavos).

Desde la abolición de la esclavitud por las fuerzas coloniales en África, a principios del siglo xx, una ambigüedad jurídica, conceptual e ideológica envuelve la práctica de la wahaya: esto trae aparejado el hecho de que el punto de vista subjetivo de estas mujeres está poco representado en las fuentes. Para remediarlo, este artículo propone que los movimientos cinéticos sean considerados como relatos: en este caso preciso, los desplazamientos fugitivos de las wahayu captan las aspiraciones de libertad y de dignidad de estas mujeres concubinas de estatus servil en el Sahel.

Las mujeres del informe mencionan en sus relatos cómo huyeron, ellas mismas $\mathrm{u}$ otras wahayu. Teniendo en cuenta las múltiples referencias a sus movilidades y a las de las demás, planteo como hipótesis que la práctica de la fuga, del cimarronaje individual en las zonas transfronterizas del sur de Níger y del norte de Nigeria es significativa y va mucho más allá de los nueve relatos grabados en el informe. El artículo analiza diversos motivos que originan el deseo de fuga de las wahayu e ilustra, con ejemplos concretos extraídos de los relatos, diversas estrategias y 
resultados de la fuga.

En conclusión, los relatos cinéticos, a saber, las fugas a través de las cuales las mujeres wahayu abandonan la casa de sus maridos/amos, son analizadas como una táctica subalterna de resistencia y de disidencia y como una aspiración a una existencia digna.

El movimiento del cuerpo en fuga es testimonio del sufrimiento prolongado que estas concubinas siguen soportando hasta hoy. En efecto, para la mayoría de las mujeres del Sahel la libertad está condicionada por el reconocimiento social de su rol de mujer y de esposa, y no por el hecho de llegar a ser individuos que disponen de una total libertad de movimiento. Pero de manera paradójica, es precisamente por el entorpecimiento de esta libertad de relación y de pertenencia, que la fuga y la capacidad individual de movimiento se vuelven para algunas la única salida a su condición.

Poucos são os relatos escritos, seja em língua vernacular seja em francês, que tratam das experiências subjetivas de exploração, de liberdade, de emancipação e de convivência dos indivíduos com status hereditário de escravo no Sahel. Os escassos documentos escritos sobre as mulheres dessa categoria foram produzidos por organizações de luta contra a escravidão, como Timidria no Níger e Temedt no Mali, ou por universitários.

Para entender a experiência subjetiva dessas mulheres, para além dos raríssimos testemunhos orais, muitas vezes enviesados, recolhidos pelos movimentos anti-escravistas, proponho sair da trilha batida das formas escritas, visuais e orais, que abundam na esfera intelectual ocidental, e considerar uma forma alternativa, composta de movimentos e de linguagem corporal: a cinética. Por « relatos cinéticos", refiro uma (i)mobilidade física atuante e corporalizada que traduz as experiências subjetivas das mulheres com status hereditário de escravo no Sahel. Analiso, mais particularmente, as suas deslocações fugitivas como táticas de resistência contra a sua exploração.

Este artigo baseia-se no exemplo específico dos movimentos de fuga das concubinas classificadas como escravas nas regiões fronteiriças do Níger e da Nigéria. Esses movimentos de mulheres são semelhantes aos marronnages dos escravos do Oceano Atlântico e do Oceano Índico. A fonte principal é um relatório baseado sobre entrevistas realizadas com concubinas por uma organização anti-escravista estabelecida no Níger. Este relatório selecionou nove casos entre 165 entrevistas, principalmente no Sul do Níger e Norte da Nigéria. Os enviesamentos decorrentes da escolha desta fonte são vários e importantes, mas são também contextualizados pelos vinte anos de experiência do autor com casos semelhantes no centro do Mali.

As wahayu - wahaya no singular - são mulheres com status de escravo que foram tomadas como concubinas por homens livres, segundo a lei islâmica. Conforme as leis abolindo a escravidão, esta prática deveria ter sido abandonada, mas não foi isso que aconteceu, antes pelo contrário. Desenvolvo a noção de pós-escravidão para explicar como a abolição da escravidão não permitiu acabar com as exclusões assentas na condição social (descendantes de senhores versus descendentes de escravos). Desde a abolição da escravidão pelas forças coloniais em África no princípio do século XX, uma ambiguidade jurídica, conceitual e ideológica manteve-se em torno da prática da wahaya: daí resulta que o ponto de vista subjetivo dessas mulheres está pouco representado nas fontes.

Para superar esse problema, este artigo defende que os movimentos cinéticos devem ser considerados como relatos: no caso aqui examinado, as deslocações fugitivas das wahayu possibilitam captar as aspirações de liberdade e dignidade destas mulheres concubinas de condição servil no Sahel.

As mulheres do relatório referem nos seus relatos como elas e outras wahayu fugiram. Considerando as múltiplas referências a sua mobilidade e a das outras, coloco a hipótese de que a prática da fuga, do marronnage individual nas zonas fronteiriças do sul do Níger e do norte da Nigéria, é significante, para além dos nove relatos registados no relatório. $O$ artigo analisa vários 
motivos que originam o desejo de fuga das wahayu e ilustra, com exemplos concretos tirados dos relatos, diversas estratégias e resultados da fuga.

Em conclusão, os relatos cinéticos, ou seja as fugas das mulheres wahayu, deixando a casa de seus maridos/senhores, são analisados como uma tática subalterna de resistência e de dissidência, e como uma aspiração a viver uma existência digna. 0 movimento do corpo em fuga testemunha o sofrimento prolongado que estas concubinas continuam a suportar hoje em dia.

$\mathrm{Na}$ verdade, para a maioria das mulheres do Sahel, a liberdade fica condicionada pelo reconhecimento social de seu papel de mulher e de esposa, e não pelo fato de tornarem-se indivíduos com uma liberdade completa de movimento. Mas, de maneira paradoxal, é justamente porque essa liberdade de relação e de pertença está sendo minada que a fuga e a capacidade individual de movimento torna-se, para algumas dessas mulheres, a única saída de sua condição.

\section{INDEX}

Palabras claves : cimarronaje, fuga, movilidad, esclavitud, relatos, Sahel, concubinas, mujeres Palavras-chave : marronnage, fuga, mobilidade, escravidão, relatos, Sahel, concubinas, mulheres

Keywords : marronage, flight, mobility, slavery, narratives, Sahel, concubines, women Mots-clés : marronnage, fuite, mobilité, esclavage, récits, Sahel, concubines, femmes

\section{AUTEURS}

\section{LOTTE PELCKMANS}

Associate professor, Centre for Advanced Migration Studies, Copenhagen University, Danemark 